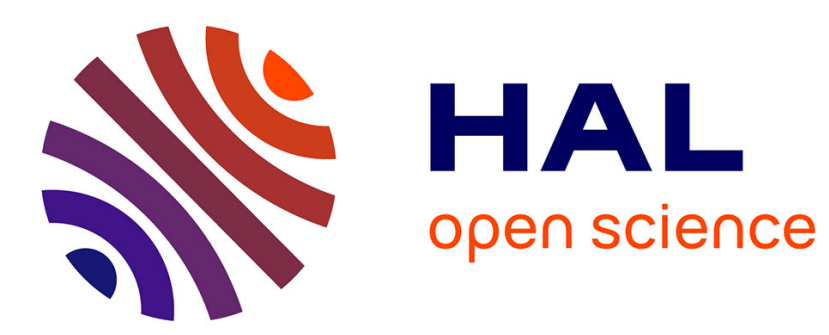

\title{
Complémentarité syntactico-énonciative entre morphèmes et intonèmes. Le cas du berbère \\ Mena B. Lafkioui
}

\section{To cite this version:}

Mena B. Lafkioui. Complémentarité syntactico-énonciative entre morphèmes et intonèmes. Le cas du berbère. Faits de langues, 2006, 27, pp.141 - 149. hal-01107485

\section{HAL Id: hal-01107485 \\ https://hal.science/hal-01107485}

Submitted on 20 Jan 2015

HAL is a multi-disciplinary open access archive for the deposit and dissemination of scientific research documents, whether they are published or not. The documents may come from teaching and research institutions in France or abroad, or from public or private research centers.
L'archive ouverte pluridisciplinaire HAL, est destinée au dépôt et à la diffusion de documents scientifiques de niveau recherche, publiés ou non, émanant des établissements d'enseignement et de recherche français ou étrangers, des laboratoires publics ou privés. 
In : A. Mettouchi \& A. Lonnet (eds), 2006, Les Langues Chamito-Sémitiques (afro-asiatiques), Volume 2, Faits de Langues, 27 : 141-149 + Références.

\section{Complémentarité syntactico-énonciative entre morphèmes et intonèmes. Le cas du berbère.}

Mena LAFKIOUI*

Notre article se veut une argumentation de l'hypothèse - née au cours de notre recherche sur l'énoncé non-verbal berbère ${ }^{1}$ - suivant laquelle il existerait en syntaxe un lien d'interdépendance (et parfois de suppléance) structurale et fonctionnelle entre les unités lexématiques (morphèmes) et les unités prosodiques (intonèmes).

Pour ce faire, nous présenterons plusieurs cas de figure provenant du tarifit, variété berbère du Maroc du Nord².

\section{LA REQUETE A STRUCTURE PREDICATIVE NON-VERBALE}

Le premier exemple présente une “prohibition ”, c'est-à-dire un acte de langage qui cherche à interdire à l'interlocuteur d'agir (verbalement ou non verbalement) d'une certaine façon. Syntaxiquement, elle est assurée par une structure fixe : modalité négative - noyau prépositionnel $/ \dot{\mathrm{g}} \overline{\mathrm{a}} /$ (ou une de ses variantes) + affixe personnel - modalité négative - complément nominal /rḥeqq/. En français, elle pourrait être traduite par “ un tel ou une telle n’a pas le droit de ...".

(01)

$$
\begin{array}{llll}
u & \dot{g} \bar{a}-\underline{k} & \underline{b u} & \text { rheqq ad } t \text {-tenged } ! \\
\text { MN } & \mathrm{P}+\mathrm{SA} & \text { MN } & \text { CO } \\
\text { ne } & \text { chez + toi } & \text { pas } & \text { droit de le+tuer }
\end{array}
$$

"Tu n’as pas le droit de le tuer !"

Cet énoncé a comme prédicat la préposition /ḡāa ('chez’). La fonction de sujetactualisateur est remplie par l'affixe personnel /-k/ ('toi'), alors que la fonction

*University of Leiden, Courriel : menalafkioui@hotmail.com

1 Voir Lafkioui (1999, 2000, 2001, 2002).

2 Les exemples examinés dans cet article proviennent d'un corpus d'oral spontané enregistré au Rif. L’analyse instrumentale correspondante a été effectuée à partir du logiciel Praat. 
de complément obligatoire est prise en charge par le syntagme /rhẹeq ad t-tenged/3 ('le droit de le tuer'). Cette prédication non-verbale est niée au moyen du marqueur de négation $/ u$... bu/. Bien qu'une intonation relativement péremptoire peut accompagner cette structure, elle n'est pas indispensable à son identification comme énoncé prohibitif. Les courbes intonatives suivantes le montrent bien :

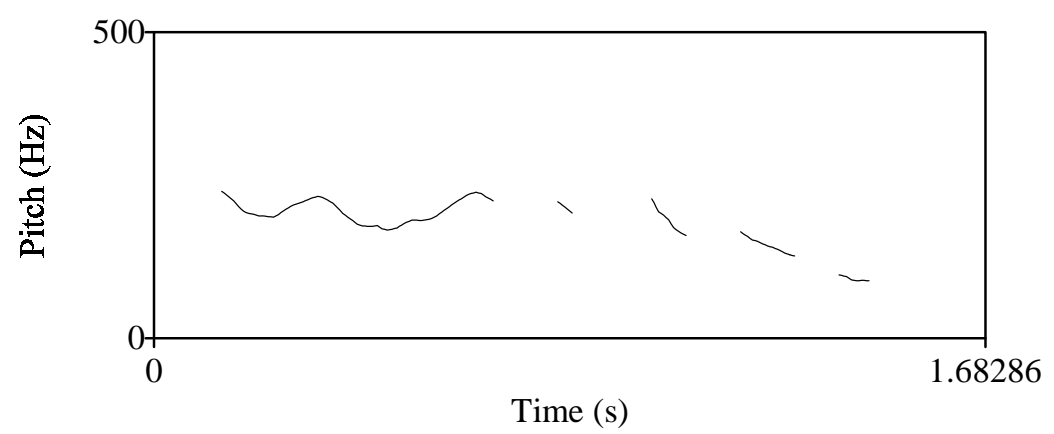

Figure 1. Contour du F0 pour l'occurrence /u gāa-k bu rheqq ad t-tenged !/"Tu n'as pas le droit de le tuer !".

Ce contour mélodique correspond au profil intonatif de l'énoncé assertif avec emphase, c'est-à-dire une courbe de type montant-descendant ${ }^{4}$ ponctuée par une montée simultanée de F0 et de I sur les deux éléments discontinus du marqueur de négation : $\mathrm{F} 0=237 \mathrm{~Hz}$ et $\mathrm{I}=93 \mathrm{~dB}$ pour le premier $/ \mathrm{u} /, \mathrm{F} 0=238 \mathrm{~Hz}$ et $\mathrm{I}=90$ $\mathrm{dB}$ pour le second. Ce pointage intonatif au niveau du morphème de négation, est à mettre en rapport avec la qualité énonciative de cette structure. Car, dans son énonciation, deux idées opposées sont mises en jeu : la première position exprime le contenu positif “ Tu as le droit de le tuer. ”. La seconde, en revanche, s'oppose à la première par le biais de l'opérateur négatif, et s'interprète donc comme “Tu n’as pas le droit de le tuer !". C’est donc la réfutation du contenu affirmatif qui entraîne la nuance sémantico-pragmatique dérivée de la prohibition.

La structure affirmative correspondante de cette prohibition est produite dans l’énoncé (02) - /ḡā-k rheqq ad th-tenġed./ ("Tu as le droit de le tuer !") - dont le schéma intonatif est :

3 Où le noyau /rhẹeqq/ est (facultativement) complété par le groupe verbal /ad t-tenged/. 4 Propre à l'énoncé assertif sans emphase, non seulement en berbère (Mettouchi \& Louali, 2002) mais aussi dans diverses autres langues du monde (Hirst \& Di Cristo, $1998: 19)$. 


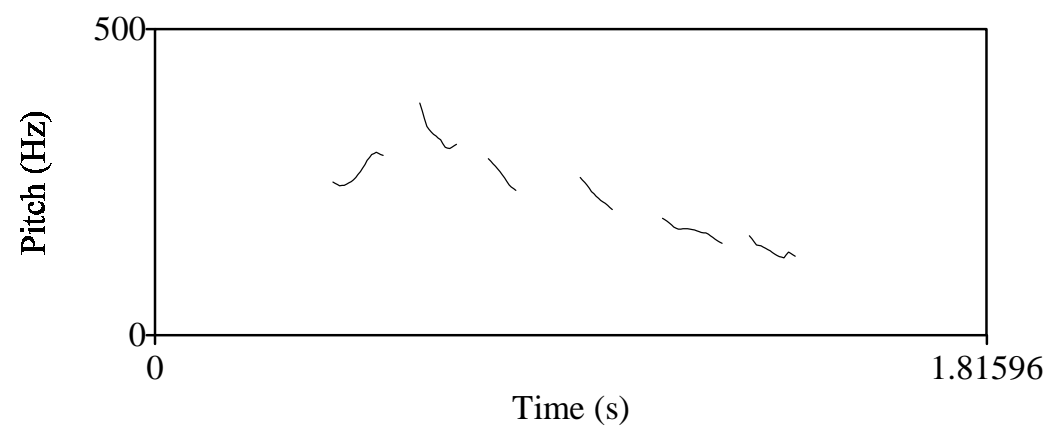

Figure 2. Contour du F0 pour l'occurrence /g்a-k rheqq ad t-tenged. / "Tu as le droit de le tuer.".

L'accent d'emphase tombe cette fois-ci sur la voyelle $/ \bar{a} /$ du prédicat prépositionnel /gàa-k/ impliquant ainsi une montée de F0 (jusqu’à $367 \mathrm{~Hz}$ ) et d'Intensité (jusqu'à $92 \mathrm{~dB}$ ).

Aucune structure intonative spécifique n’est donc réservée à l'énoncé nonverbal prohibitif. Mais, c'est la manifestation de certains morphèmes et leur agencement selon un ordre bien précis qui l'identifie en tant que tel.

La requête suivante est de type " avertissement " : l'acte de langage par lequel le locuteur essaie de donner un avis à l'allocutaire sous forme d’une prévention.

$$
\begin{aligned}
& \text { gā-m! } \\
& \mathrm{P}+\mathrm{SA} \\
& \text { chez + toi } \\
& \text { "Fais attention!" }
\end{aligned}
$$

Cet énoncé présente la structure prototypique d’un avertissement à centre syntaxique non-verbal. Celui-ci est assumé ici par la préposition /ġā/. Il constitue avec le sujet-actualisateur /-m/ le syntagme prédicatif. Tous deux forment une structure syntaxique adéquate (nucléaire) qui répond, grâce à son intonation et son contexte appropriés, aux critères de la validation sémantico-énonciative. Sans le facteur contextuel et encore davantage le facteur prosodique, cette structure demeure incomplète, car l'agencement minimal d'un énoncé à prédicat prépositionnel exige en principe la présence d'un complément obligatoire (Lafkioui, 1999). Sur le plan intonatif, ces deux courbes sont possibles : 


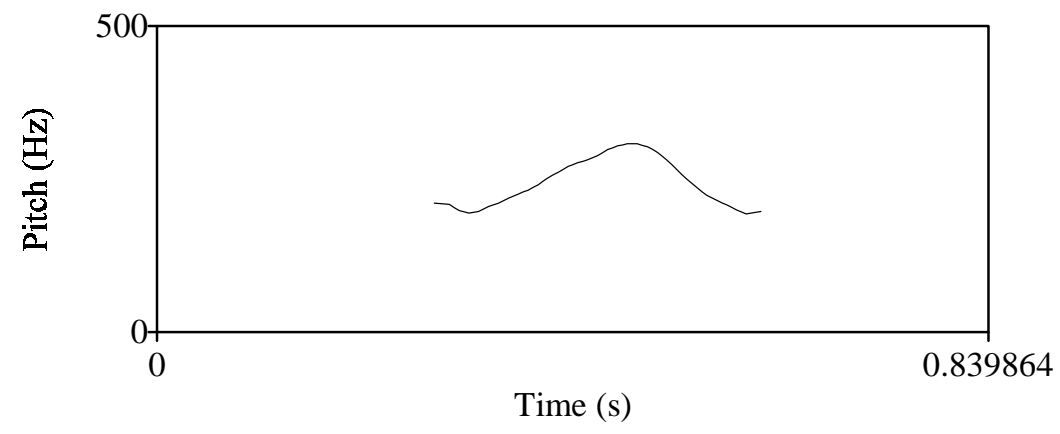

Figure 3. Contour du F0 pour l'occurrence /ġā-m !/"Fais attention !", énoncé dans un contexte de danger.

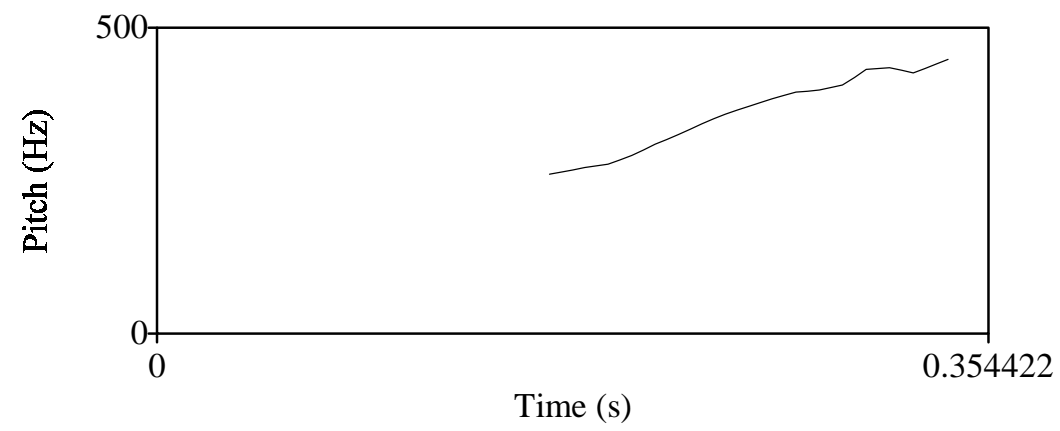

Figure 4. Contour du F0 pour l'occurrence /ġā-m !/"Fais attention !", énoncé dans un contexte de danger imminent.

Toutes deux connaissent, dès le début de la syllabe, une montée rapide de la force fondamentale et de l'intensité. Dans la Figure 3, F0 change de direction une fois passée par son sommet qui coïncide avec la voyelle /âa/. La fondamentale de la dernière courbe, par contre, continue sa montée croissante sans déclin et atteint son point culminant à la fin de la syllabe dans un lapsus de temps relativement court : $354 \mathrm{~ms}$ par rapport $840 \mathrm{~ms}$. Ce schéma intonatif (F0+, I+, D-) semble être caractéristique pour l'exclamation berbère, ce qui pourrait nous amener à classer ce type d'énoncé dans la catégorie fonctionnelle des exclamations. Néanmoins, nous préférons le ranger parmi les requêtes, parce qu'il met clairement en évidence le désir du locuteur de faire accomplir un certain acte verbal ou non-verbal. D'autant plus que cette structure prépositionnelle - qui semble au premier abord être (syntaxiquement) 
défectueuse - apparaît uniquement dans un contexte fonctionnel d'avertissement. Ceci se reflète clairement dans son sens de base que nous pourrions traduire en français par "Fais attention !" ou "Faites attention ! ”, selon le genre et le nombre du sujet-actualisateur.

Il importe de signaler le rôle primordial du contexte immédiat dans le choix des intonèmes et de leur conjonction. Le danger imminent ressenti par le locuteur de l'énoncé de la Figure 4 dicte nécessairement une intonation très appellative, voire même contraignante, afin que le locuteur puisse voir son acte de langage réussir.

Nous avons également constaté que, dès qu'on rajoute un complément obligatoire à cette configuration nucléaire, il se produit un changement au niveau du centre d'intérêt du locuteur. Ce n'est plus l'expression d'un éventuel danger mise en mots par le groupe prépositionnel et soutenue par l'intonation - qui intéresse le locuteur, mais le souhait de convaincre l'allocutaire de prendre soin de l'objet ou de la personne nommée par le complément obligatoire. L'exemple suivant en fait témoignage :
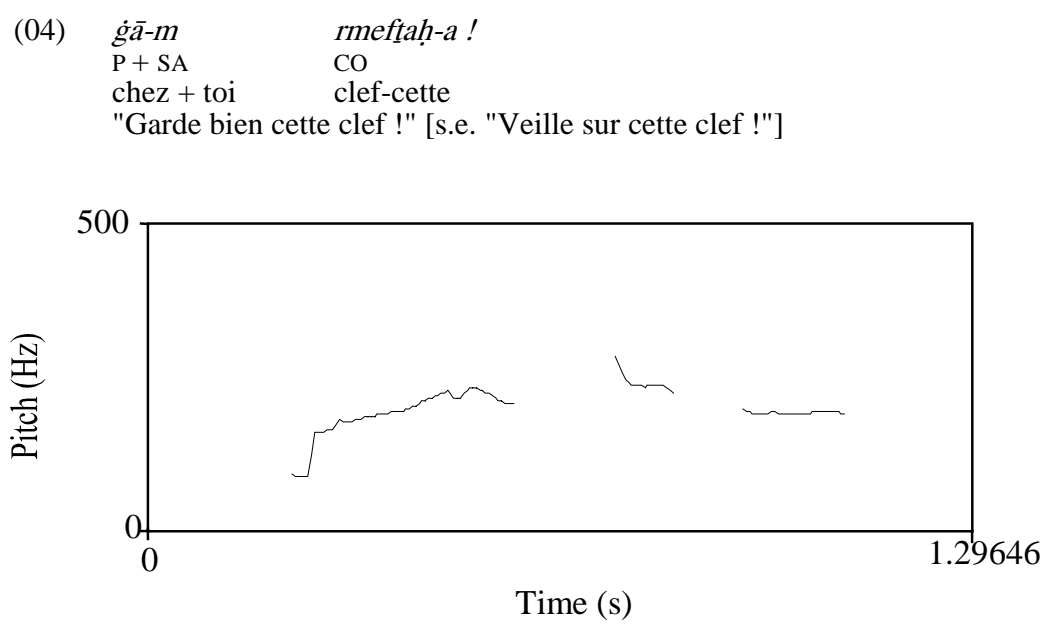

Figure 5. Contour du F0 pour l'occurrence /ḡā-m rmeftah-a !/"Veille sur cette clef!".

Ce déplacement de l'objet d'intérêt du locuteur est intonativement signalé et corroboré par l'association du summum mélodique avec l'avant-dernière syllabe du complément /rmeftah-a/. Remarquons que le trajectoire intonatif de cet énoncé est semblable à celui de l'avertissement (03) tel qu'il est présenté dans la Figure 3. Cette intonation d'avertissement - étant la seule intonation compatible avec cette structure morphématique - est essentielle à la validation syntaxique et 
sémantique de cet énoncé. Cette contrainte de "syntaxique intégrée "5 est principalement induite par la présence du morphème démonstratif /-a/ (" cette "). Il réserve cette structure exclusivement à la requête exprimant un avertissement. L'omission de ce démonstratif rendrait à nouveau la structure accessible à d'autres qualités énonciatives, telles que l'assertion ou la question. Et c'est l'intonation qui décidera, dans ce cas, de quel type d'énoncé il s'agit. Ainsi, la construction de départ /ḡà-m rmeftah/ présente, selon l'intonation avec laquelle elle se conjugue, une assertion (Figure 6 et Figure 7), une question (Figure 8) ou une requête (Figure 9).

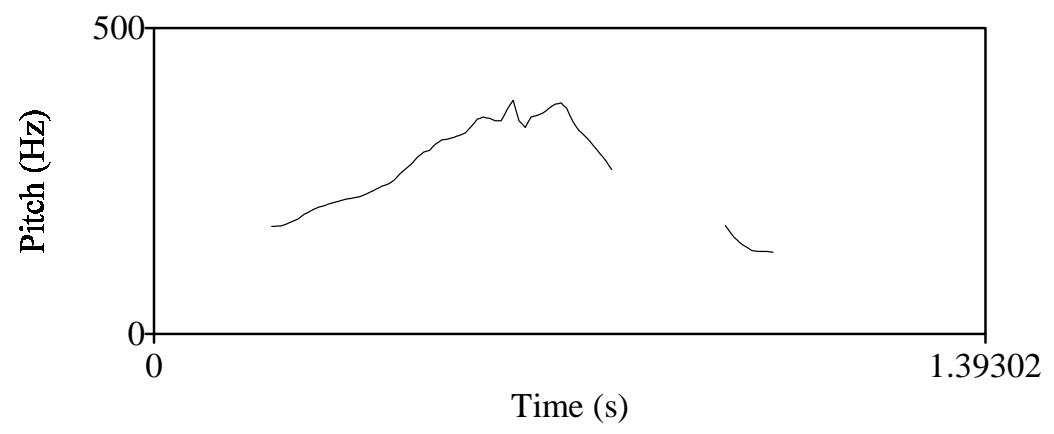

Figure 6. Contour du FO pour l'occurrence /g்à-m rmeftah.l/"(Mais) tu as une clef".

5 Approche mettant en valeur l'idée que la syntaxe, la sémantique et la pragmatique se conjuguent intégralement et de façon continuelle (voir Lafkioui, 1999, 2000, 2001, 2002) 


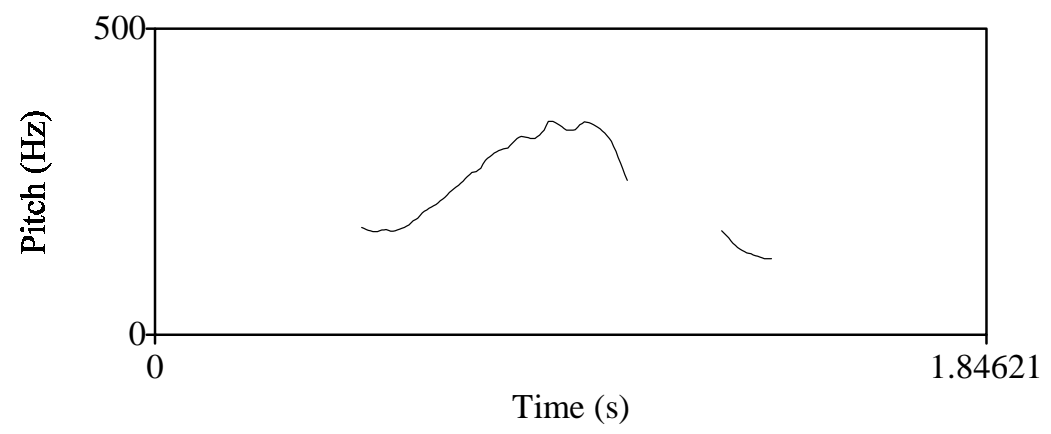

Figure 7. Contour du FO pour l'occurrence /qa ġā-m rmeftah./"Mais tu as une clef".

Dans la Figure 7, le message asserté - “Mais tu as une clef.” servant de réplique à une demande de prêt de la clef du locuteur - est épaulé par la présence du marqueur discursif /qa/. Celui-ci ne permet aucune articulation intonative autre que celle de l'assertion. La performance de ce morphème l'emporte donc ici sur l’intonation.

Lorsque les morphèmes / $\dot{\mathrm{g} a}-\mathrm{m}$ rmeftah/ se conjuguent avec une intonation dont F0 monte progressivement jusqu'à la dernière syllabe de /rmeftah/ (voir cidessous Figure 8), l'ensemble acquiert le statut de question à condition qu'il soit énoncé dans un contexte d’interlocution.

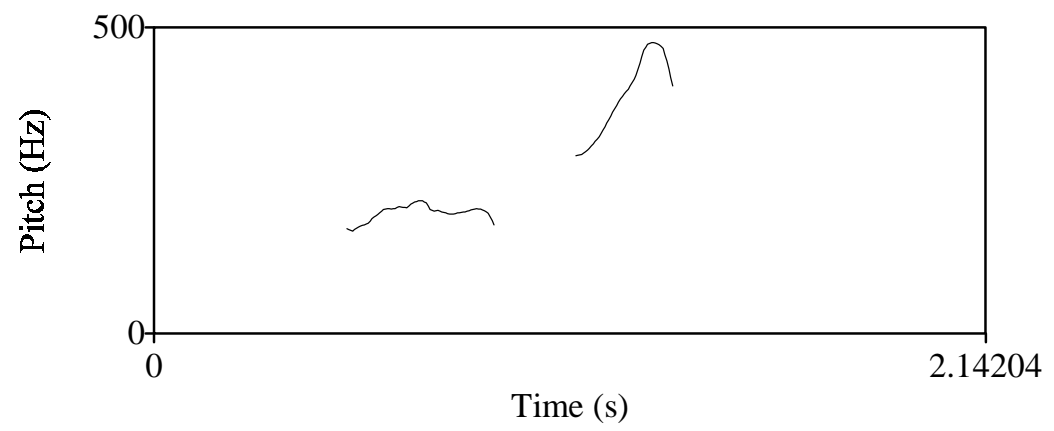

Figure 8. Contour du F0 pour l'occurrence /ḡā-m rmeftah ?/"Tu as une clef ?". 
Le profil intonatif de l'avertissement /ġā-m rmeftạ !/, présenté dans la Figure 9, est comparable à celui des autres avertissements traités antérieurement dans cet article. Il s'agit d'une montée significative (et relativement rapide) de la force fondamentale et de l'intensité dès la première syllabe de l'énoncé, suivie éventuellement d’une chute mélodique une fois le pic passée.

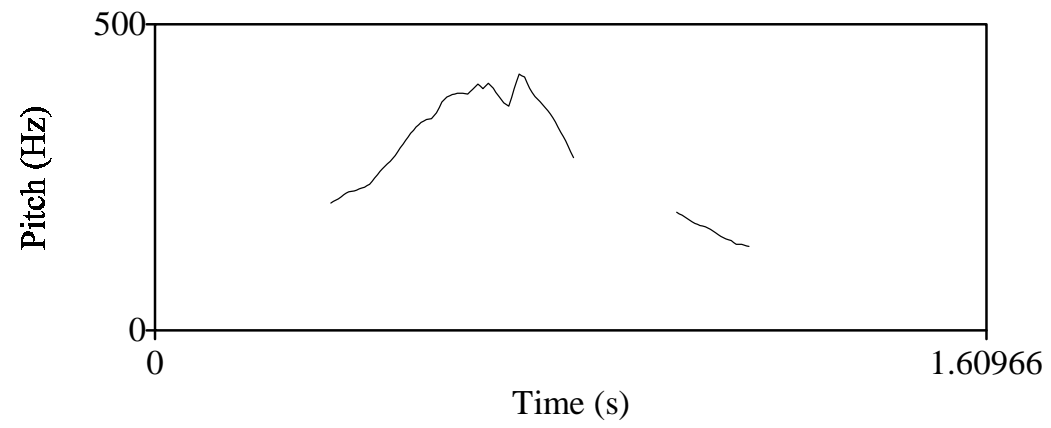

Figure 9. Contour du FO pour l'occurrence /ġā-m rmeftah! !/ "Veille sur la clef!".

Outre les traits intonatifs $\{\mathrm{F} 0+, \mathrm{I}+, \mathrm{D}-\}$, cet exemple fait clairement paraître que l'avertissement se distingue surtout par le niveau d'hauteur mélodique. Sa valeur maximale $(420 \mathrm{~Hz})$ dépasse nettement ce qu'on retrouve par exemple dans l'assertion /qa ġā-m rmeftah./ (Figure7 : F0max $=350 \mathrm{~Hz}$ ) qui témoigne d'un schéma intonatif analogue.

\section{LES ASSERTIONS NON-VERBALES A JUXTAPOSITION DE DEUX NOMINAUX.}

La distribution syntaxique de ce type d'assertion est fixe et répond au critère de bi-fonctionnalité. Elle se décrit généralement comme suit : NOM1 = sujetactualisateur - marqueur intonatif - NOM2 = prédicat. La position syntagmatique forme donc un critère important pour l'identification de ces fonctions syntaxiques de base. Cependant, leur démarcation syntaxique repose principalement sur des critères intonatifs, qui sont d'ailleurs les seuls à pouvoir faire une distinction entre un énoncé complet (05) et un syntagme appositif (06).

(05)

\begin{tabular}{lll} 
ah̆āmuš & \multicolumn{1}{l}{$I-$} & amzyan \\
S & MI & P \\
garçon & petit & \\
"Le garçon est petit." &
\end{tabular}



(06) ahāmuš amzyan NDE NDANT
Garçon petit
"Le / un petit garçon"

Les noms de ces exemples - identique sur le plan morphologique - constituent, à l'aide d'une intonation appropriée, une assertion finie (Figure 10) ou bien un simple syntagme déterminatif de type déterminé-déterminant (Figure 11).

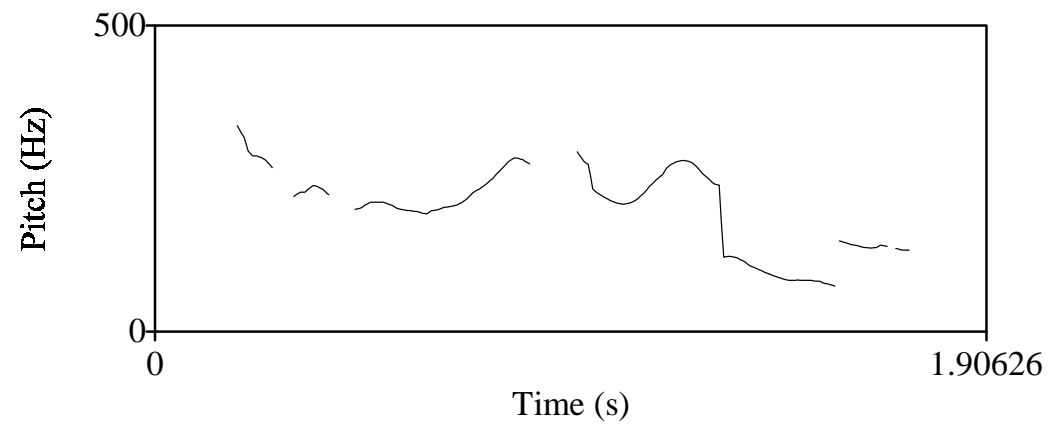

Figure 10. Contour du F0 pour l'occurrence /aḥāmuš \{I-\} amzyan./"Le garçon est petit.".

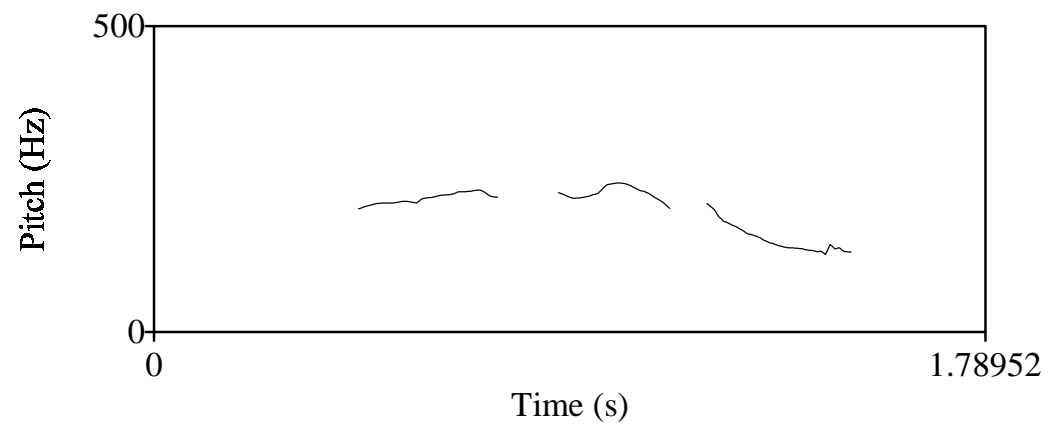

Figure 11. Contour du FO pour l’occurrence /ahāmuš amzyan./ "le petit garçon". 
Non seulement la forme des courbes de F0 diffère dans les deux cas, mais aussi leur schéma d'intensité correspondant, surtout au niveau de la frontière entre NOM1 et NOM2. Dans l'énoncé (05) [Figure 10], on passe d'une intensité de 88 $\mathrm{dB}$ sur la voyelle /u/ de la dernière syllabe de NOM 1 à une intensité de $76 \mathrm{~dB}$ sur la voyelle /a/ de la première syllabe de NOM2. Ceci explique la fausse impression d'un décrochage mélodique que l'on a à l'ouïe, puisque F0 évolue à ce niveau dans la même plage intonative : respectivement $284 \mathrm{~Hz}$ pour /u/ et 289 $\mathrm{Hz}$ pour /a/. La progression de l'intensité dans l'exemple (06) [Figure 11], par contre, ne connaît pas de chute : les deux valeurs tournent autour de $87 \mathrm{~dB}$.

Ainsi, ces analyses portent appui à la thèse de F. Daneš (1960) suivant laquelle la fonction fondamentale de l'intonation est de transformer des lexèmes en énoncés :

" The fundamental function of intonation is to transform words, as appelative units, into communicative units, i. e. into utterances. ”.

\section{Références bibliographiques}

Daneš F., 1960, Sentence intonation from a functional point of view, WORD 16, p. 34-54.

Hirst D. \& Di Cristo A. (eds), 1998a, Intonation Systems : a Survey of Twenty Languages, Cambridge, Cambridge University Press.

Lafkioui M., 1999, Syntaxe intégrée de l'énoncé non-verbal berbère, Thèse de Doctorat (dir. S. Chaker), Paris, Inalco, tome I (416 p.) + tome II (annexe corpus).

Lafkioui M., 2000, Syntaxe intégrée de l'énoncé non-verbal berbère : l'énoncé à auxiliaire de prédication spécifique, Comptes rendus du G.L.E.C.S. XXXIII, Paris, p. 165-187.

Lafkioui M., 2001, Typologie des assertions affirmatives non-verbales du rifain. Approche de syntaxe intégrée, Frankfurter Afrikanistische Blätter 13, p. 97120.

Lafkioui M., 2002, L'intonation et ses fonctions syntaxiques en rifain, in Articles de linguistique berbère. Mémorial Werner Vycichl, K. Naït-Zerrad (ed.), Paris, L'Harmattan, p. 253-281.

Mettouchi A. \& Louali N., 2002, Structures intonatives en berbère : l'énoncé prédicatif à particule d, in Bel B. \& Marlien I. (eds), Aix-en-Provence, p. 463-466. 